also at 2 weeks from injury compared with healthy control children.

Methods Whole blood was sampled from children with mild TBI within 24 hours of injury and at two weeks from injury and compared to healthy paediatric controls at baseline. RNA was isolated and cDNA was synthesized. Gene Expression of NLRP3 and IL1 $\boldsymbol{\beta}$ via rtPCR was recorded in 22 patients and 5 controls at baseline and 15 patients at 2 weeks. The Post Concussive Symptom Inventory was administered at 2 weeks. A change from pre-injury baseline was recorded.

Results Inflammasome was upregulated via NLRP3 expression in children with TBI compared to controls across groups however this did not correlate with symptoms at 2 weeks. Higher IL-1 $\beta$ transcription levels at presentation were positively correlated by Pearson correlation $(\mathrm{p}=0.029)$ with higher symptom scores at 2 weeks.

Conclusion Inflammation is altered in TBI compared to controls The NLRP3 component of inflammasome while elevated does not correlate with symptom burden. $\mathrm{IL}=1 \boldsymbol{\beta}$ gene transcription does. IL-1 $\boldsymbol{\beta}$ holds promise in predicting symptom burden following mTBI. Selective inhibition of systemic inflammation targeting the inflammasome may have a future immunomodulatory role as a target in treating mTBI.

\section{GP125 MY LIFE, MY VOICE: TECHNOLOGY-ENHANCED QUALITY OF LIFE ASSESSMENT TOOL FOR CHILDREN WITH HYDROCEPHALUS}

1,2,3 Joy Tan*, ${ }^{3,1}$ John Caird, ${ }^{1,2}$ Alf Nicholson. ${ }^{1}$ Temple Street Children University Hospital, Dublin, Ireland; ${ }^{2} R C S I$, Dublin, Ireland; ${ }^{3}$ Beaumont Hospital, Dublin, Ireland

\subsection{6/archdischild-2019-epa.190}

Background To date, children with hydrocephalus continue to have a considerable long-term outcome. However, current literature on health-related quality of life (HRQOL) among children with hydrocephalus are limited. This serves a call for research to validate a suitable HRQOL for children with hydrocephalus measuring the physical, emotional, social and cognitive well being.

Objective To validate a technology-enhanced quality of life questionnaire (EITVAQ) as an effective assessment tool measuring the current well-being of a child with hydrocephalus (child-centred)

To adopt a technology-enhanced quality of life assessment tool (EITVAQ) into a phone/tablet application to gain widespread usage

To explore the use of technology to increase the learning potential and obtaining a new skill among children with hydrocephalus

Methods This is a prospective study which took place since January 2018 to January 2019.

This study received ethical approval All data was compiled and analysed using SPSS. The validation process involved: Content Validity, Feasibility and Reliability. A cohort of 70 healthy children from various primary and secondary schools participated as a control. Finally, a small cohort of children with hydrocephalus participated in technology-enhanced learning programme (TELP) to learn a new music skill and created a music video.
Results This study reports the result of the validation process. In total there were 132 participants. Content validity was achieved by the nominal group technique. In this study, technology enhanced quality of life questionnaire (EITVAQ) is a feasible tool, having a high satisfaction rate of $78 \%$, a response rate of $76.5 \%$ and an average completion time of 4 mins and 26 seconds. EITVAQ had a score of 0.92 (Cronbach Alpha). When comparing with the control, the two lowest scores were the social and emotional aspect. The two major differences in scores among both groups were the physical and cognitive well-being. All participants who participated in the TELP managed to complete the task learning the skills of creating a music video.

Conclusion EITVAQ, an interactive and child-friendly tool to assess quality of life is now validated. It aims to be used widely among children with hydrocephalus, providing a baseline assessment to allow us to understand more about a child's quality of life from their own perspective. This study concludes that technology has a huge potential in helping children with hydrocephalus and various neuro-disability to integrate into society.

\section{GP126 LARGE DELETIONS IN DMD GENE IS THE MOST PREVALENCE MUTATION IN RUSSIAN CHILDREN WITH DUCHENNE MUSCULAR DYSTROPHY}

Kirill Savostyanov*, Alexander Pushkov, Ludmila Kuzenkova, Natalya Zhurkova, Alexey Nikitin, Alexey Kurenkov, Bella Bursagova, Sergey Trufanov. FSAI National Medical Research Center for Childrens Health, Moscow, Russian Federation

\subsection{6/archdischild-2019-epa.191}

Background and objectives Duchenne muscular dystrophy (DMD) is a rare muscle disorder inherited by X-linked recessive type and affecting approximately 1 in 3,500 male births worldwide.

Patients and methods The study included 63 boys, aged from 6 months to 8 years with elevated creatinine phosphokinase (CPK), according to laboratory tests. After medical genetic counseling molecular genetic analysis was performed for all patients. The MLPA method was used to search for deletions and duplications in the $D M D$ gene, the analysis of point mutations was carried out by NGS, if the MLPA method did not reveal pathogenic variants. Sanger sequencing was used to validate mutations identified by the NGS.

Results Totally, in 39 patients we revealed different alterations in DMD gene. Among them 11 (28\%) had a point mutations. It was 4 nonsense, 4 missense, 2 splicing mutation and one single-nucleotide duplication. Five mutation were novel. They are splicing c.10798-2A>G, missense c.2288T $>A$ (p.Val763Asp) and c.3269A >T (p.Gln1090Leu), nonsense $c .858 T>G(p . T y r 286 X)$ and duplication c.8325dup (p.Gln2776Thrfs*6).The remaining $28(72 \%)$ patients had gross duplications $3(8 \%)$ and gross deletions $25(64 \%)$ in the $D M D$ gene. Interestingly, more than half of the patients had deletions in the region of exons 45-51 of the DMD gene.

Conclusion Our study showed that the most common cause of Duchenne dystrophy in Russian children are gross deletions of the $D M D$ gene, in particular deletions in the region of exons 45-51 occurring most frequently. 